\title{
Teaching Research Methodology: Everything's a Case
}

\author{
CD Reddy \\ University of Johannesburg, Auckland Park, South Africa \\ creddy@uj.ac.za
}

DOI: 10.34190/JBRM.18.2.009

\begin{abstract}
This paper contributes to the discourse about research methodology pedagogy towards the development of new scholarly researchers. Because students can better visualise causal effects within a single case, I propose that instructors, before moving onto to teach inferential statistics relying on impersonal numbers from many cases, use instead a caseoriented approach to emphasise the "case" as the basic pedagogic unit to scaffold the student's learning of research methodology. A carefully chosen case can represent an instantiation of the same phenomenon that will eventually be used to demonstrate inferential statistics. After all, for causal effects to show up at the population level, they must be operating within particular cases. I refer to this as an $\mathrm{N}$-case approach. This approach locates various combinations of quantitative and qualitative methods on a continuum from exploratory to confirmatory research. This continuum can also be framed as one moving from the particular to the general. The single case allows students to grasp ideas about causal processes in a more direct manner than a survey can. Instructors can then build on single case insights to grasp similar ideas at a population level. My motivation for this approach is to turn away from a pedagogy where research methodology teaching begins with ideas set in quantitative approaches to understand causal processes occurring at a particular case level. I also believe that current research teaching ought to turn away from encouraging students to apply a research design according to their favoured identity along a qualitative-quantitative divide. Instead, the research problem should determine the required research design.
\end{abstract}

Keywords: research design, research method, qualitative, quantitative, pedagogy, teaching, case study

\section{Introduction}

How can we teach research methodology at graduate level? A useful way to design research methodology courses is to align them with the process of knowledge discovery and, in particular, theory building (Edmondson and McManus, 2007). After all, many students who go on to become scholars will continue to evaluate existing theories and even build theories; and those who become management practitioners will find their theory building skills useful to interrogate the assumptions of their management decisions gone awry. But rather than align research methodology teaching with the reality of the theory building process, we instructors follow textbooks, which tend to be divided into quantitative and qualitative methods. And in these textbooks, the content devoted to quantitative methods outweighs that devoted to qualitative methods (Onwuegbuzie and Leech, 2005). Yet the qualitative method, applied to a single case or small number of cases, can serve to better illustrate to students the beginnings of theory development.

Recognizing this dilemma, scholars propose a mixed methods approach that delineates research into exploratory and confirmatory techniques (Onwuegbuzie and Leech, 2005). This approach models the reality of researchers having to gather both qualitative and quantitative data to answer their research question. The approach has gained favour because it demonstrates to students the complementary nature of qualitative and quantitative methods (Christ, 2009; Earley, 2007; Hesse-Biber, 2015; Ivankova and Clark, 2018; Onwuegbuzie, Frels, Collins and Leech, 2013; Tashakkori and Teddlie, 2010). In order to reduce statistics anxiety among students, scholars propose that this approach be scaffolded so that exploratory type research courses, with qualitative analysis techniques and descriptive type statistics, are delivered before confirmatory type research courses with more complex probabilistic type statistics (Onwuegbuzie, Leech, Murtonen and Tähtinen, 2010a).

Building on this exploratory-confirmatory continuum, I propose a case-oriented approach to teach research methodology. This approach uses the "case" as the building block and unifying pedagogical unit. When researchers explore a phenomenon through a single case, apart from the traditional qualitative data available from participant narratives, they also come across important quantitative data. Note too, for causal effects to show up at the population level, they must be operating within particular cases explored by researchers. Putting a name to this model, I refer to it as an $\mathrm{N}$-case approach. This approach involves designing research methodology courses and teaching them in sequence from a single case study to small-to-medium- $\mathrm{N}$ case designs and finally to large- $\mathrm{N}$ case designs where students get to learn complex inferential and probabilistic statistical techniques. If we can first expose students to explanatory narratives and causal effects occurring within a single case, we 
might go some way to help them overcome their statistics anxiety when they learn to apply statistical techniques to confirm whether a case-level explanation applies to large- $\mathrm{N}$ cases.

This article will develop the idea of teaching research methodology on a continuum (Onwuegbuzie and Leech, 2005). It adopts Onwuegbuzie et al's (2010a) matching of various analytical techniques to this continuum. In attempting to fulfil an overall explanatory objective, we will learn that a single or a small number of cases can fulfil an exploratory research objective and a medium to large number of cases can confirm if the emerging explanation applies generally. This approach responds to recent calls to scaffold students learning experience of research methodology (Ivankova and Clark, 2018). Students can better visualize the explanatory logic behind a particular phenomenon when presented with a tangible story. This story is best distilled from the process narrative of a single case of the phenomenon rather than several cases occurring as impersonal numbers and words from survey data. The case can represent behaviour at an individual, firm, group or societal level. The case can also be an instantiation of an intervention as in action research; or an instantiation of group behaviour as in an ethnography. Ultimately, I hope that this way of teaching can help train new researchers to transition from developing narrow empirical explanations of phenomena to developing explanations at a broad theoretical level. Next, I elaborate on the theoretical background for my position and then I present ideas for implementing the $\mathrm{N}$-case approach before discussing the implications of this approach for research methodology teaching and practice. Because this approach is yet to be tested in a classroom, I have no empirical evidence to report; and thus this attempt can be treated simply as a proposal. My hope is for research methodology scholars to design action research around teaching interventions involving this $\mathrm{N}$-case approach.

\section{Theoretical Background}

Particularly, for graduate research, we teach students about how to develop a research problem founded on some situation in the real world and to solve this problem by either building a new theory or modifying an existing theory, often in a small but significant way. But what is a theory? In the field of business management, a theory is a chosen way to explain a certain outcome of organizational or management behaviour. The theory goes on to identify the antecedent conditions and boundary conditions of the outcome, how they lead to the outcome and why they lead to the outcome. Put another way, a theory includes the what, the 'who, when and where', the how and the why (Whetten, 1989). In symbolic form, we can represent this as follows (see Gerring, 2007):

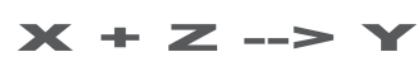

In this framework, $X$ can include more than one antecedent condition e.g. $x 1, x 2$ and $x 3$ and $Z$ can include more than one background condition e.g. $z 1$ and z2. These $z$ factors represent the "who, when and where" conditions. The $Y$ represents the outcome. Together with the symbols representing the what, who when and where, a theory also involves a narrative about how and why the $X$ and $Z$ factors lead to $a Y$ outcome. The latter involves narrating how $X$ is necessary and/or sufficient for $Y$ to occur. Thus, it is important to realise that the statistical coefficient that represents the effect size of the $X$ variable is not what makes it a cause. The effect size is merely a symptom of it being a necessary and/or sufficient condition for $Y$ to occur (Mahoney, 2008). To "see" and understand the dynamics of a causal effect one needs to delve into one or a few units or cases of the phenomenon.

Notably, before one develops explanatory type research objectives, one relies on prior exploratory research or, when this does not exist, one designs exploratory research to make meaning of and delineate a phenomenon including its outcomes and their respective conditions. This interpretive goal is important because without agreeing on the meaning of various aspects of a certain phenomenon, one cannot easily agree on its explanation (de Vaus, 2001). Researchers often begin with a single case of a certain phenomenon to explore its meaning or current interpretation. This case study is "an empirical inquiry that investigates a contemporary phenomenon within its real-life context; when the boundaries between phenomenon and context are not clearly evident; and in which multiple sources of evidence are used" (Yin, 1984:23). Moving on from interpretive goals, researchers have not leapt into large-N surveys; instead they have been using multiple case studies to also fulfil positivist goals; but research pedagogy has yet to catch up to this research practice (e.g. Eisenhardt, 1989; Ragin, 2014).

Textbooks have tried to capture this research process through a delineation into quantitative and qualitative approaches. But in actual research practice, methods do not possess the clear boundaries depicted in textbooks. The names given to certain methods merely serve to contribute towards a classification system for instructional and analytical purposes and often becomes unhelpful to research instruction and practice because there is no 
fixed way of ordering a research inquiry. Take for example an ethnography-- a case study of a group of people who share some behavioral characteristics--which textbooks categorize as a qualitative research approach but in practice can 'entail both qualitative and quantitative data collection and analysis' (Sandelowski, 2010: p81).

As a result of their training, new "quantitative" researchers risk assuming that explanations of a certain phenomenon can best be understood in a probabilistic fashion; and new "qualitative" researchers risk assuming that explanations can be understood in a deterministic fashion through the presence of necessary and sufficient conditions (Beach and Pederson, 2013). But both approaches lie on the value chain of theory building: one helps to develop the theory and the other helps to establish if the theory applies more generally. The unit of analysis that is common throughout this value chain is the "case", which can be analysed individually, in groups or as an instance of a certain phenomenon. For example, there can be a case of an individual or the case of a household or the case of an organisation or the case of unethical business behaviour.

A single case about a certain phenomenon can reveal something in part about an entire population of cases (Gerring, 2007). For example, a process tracing exercise on a single case can begin to reveal the bare bones of an explanation about how and why $\mathrm{X}$ leads to $\mathrm{Y}$. One gets to observe that changes in $\mathrm{X}$ can lead to changes in $\mathrm{Y}$.

This knowledge can be used as the building block to develop a student's understanding of regression analysis. It can also introduce the idea of variance over time, which is a useful building block to teach longitudinal or panel analysis. But, before leaping to regression analysis of a large number of cases, one can add a few cases to the original case to introduce the idea of variance across cases and techniques of comparative or "between-case" analysis. This comparative analysis affords instructors the opportunity to teach the logic of Mills methods of difference and agreement.

In this manner, instructors can demonstrate how one can use small-N case designs to test propositions. However, these are not propositions about average independent effects or about the constant conjunction of cause and effect in a population. Instead, small-N case designs involve the evaluation of propositions about the presence or absence of certain conditions on the causal pathway. Importantly, because one can observe conditions, at a case level, varying and showing up as effects when examined in large-N research, we can retain the same case-level causal language for large-N quantitative studies (Mahoney, 2008).

Fortunately, over the years, there has been an increase in books showing how one can use a single case to study a contemporary phenomenon within its real-life context and go on to use variable-centred logic to relate the antecedent conditions of the phenomenon to abstract theoretical concepts (Blatter and Haverland, 2012; Hall, 2003; Van de Ven, 2007). This has demonstrated the ease with which one can navigate back and forth between interpretive and positivist perspectives. In fact, many researchers have taken an explicit positivist approach to case studies, emphasising their goal to use a case study to develop a theory that can be applied to an entire population of cases (Gerring, 2007; King Keohane and Verba, 2007). Earlier, Mahoney (2008) attempted to develop a unified theory of causality. In this theory he argues that the causal effects shown as "statistical likelihood that operates probabilistically within a population" represents the same causal process easily visualised at a case level (p.413). For causal effects to show up at the population level, they must be operating within particular cases. In other words, these effects do not suddenly emerge; they can indeed be reduced to the processes that occur within a particular case. In fact, "case-level causation is ontologically prior to population-level causation" (Mahoney, 2008: p414).

Evidently, this bottom-up approach of building a causal argument has yet to reach our research methodology pedagogy. Instructors continue to develop causal models at the population level and then use this foundation to teach causal processes at a case level. Below, I attempt to show an approach in which we can begin to teach research methodology by developing a substantive theory from observing causal processes at the level of a particular case and then using this substantive understanding as a building block to explain how the theory is more generally applicable at a population level. Students will find it simpler to develop a mental picture of covariational observations from single or small-N cases and this can serve as a useful building block to understand co-variational thinking within complex statistical techniques used for large- $\mathrm{N}$ studies. 


\section{Teaching Application}

Table 1 presents an $\mathrm{N}$-case approach. $\mathrm{N}$ refers to the number or quantity of cases. It's a tall order trying to develop a "capture all" label for single case" (i.e., with $\mathrm{N}=1$ ), small-N, medium- $\mathrm{N}$, and large N-cases. Generally the label multiple case (Eisenhardt, 1989) has been used to refer to the small-N case design. On the other hand, the qualitative comparative analysis (QCA) methods (see Ragin, 2014) can encompass both small-N and medium$\mathrm{N}$ case designs but QCA texts tend to focus on more than ten cases but not in excess of fifty. Large N-cases, as we know, are synonymous with a survey. Of note, I use the descriptor "case" as a synonym for "unit." More generally, cases can be considered as the units of analysis; 'variables' as characteristics of cases and 'observations' as the number of times each case is observed (see King et al, 2007).

Table 1 delineates research methodology into four courses: first, an introductory course to describe the theory building process along an exploratory-confirmatory continuum; second, a course illustrating a single case design and method; third, a course illustrating research design and methods with small to medium number of cases; and finally, a course illustrating a research design and methods with large number of cases. To reiterate, I begin with the single case because, unlike large-N cases, it helps students "listen" to particular actors, and to "see" their characteristics and activities enroute to an outcome of interest (Blatter and Haverland, 2012).

This framework retains the qualitative and quantitative terminology; but, this time, as types of data and analytical techniques. Notably, both types of data can be used to explore a phenomenon (Onwuegbuzie and Leech, 2005) but analytical techniques must be deployed along the exploratory to confirmatory research continuum. Thus Onwuegbuzie et al (2010a) propose redesigning research methodology courses according to exploratory and confirmatory research techniques where students learn how both quantitative and qualitative data can be used simultaneously. On the one hand, an exploratory course can focus on quantitative analyses such as descriptive statistics and qualitative analyses such as coding, keywords-in-context, content analyses and method of constant comparison. Advanced exploratory courses can focus on quantitative techniques such as factor and cluster analyses and qualitative techniques such as domain analysis, taxonomic analysis and componential analyses. On the other hand, a confirmatory course can focus on the probabilistic quantitative techniques such as univariate inferential statistics and qualitative techniques such as axial coding. More advanced confirmatory courses can focus on probabilistic quantitative techniques such as multivariate inferential statistics and qualitative techniques such as selective coding, qualitative comparative analysis and semantic network analysis (Onwuegbuzie et al, 2010a: p36).

Table 1: N-case approach to research methodology teaching at graduate level

\begin{tabular}{|l|l|l|l|}
\hline $\begin{array}{l}\text { Proposed } \\
\text { courses }\end{array}$ & $\begin{array}{l}\text { Level of } \\
\text { knowledge } \\
\text { from prior } \\
\text { research }\end{array}$ & Research Purpose & Learning Content/Outcomes \\
\hline $\begin{array}{l}\text { Course 1: } \\
\text { Scholarly }\end{array}$ & & Describe the theory building process & $\begin{array}{l}\text { Theory construction } \\
\text { Induction-deduction cycle } \\
\text { Toulmin argument } \\
\text { Scientific enquiry } \\
\text { Relationship between theoretical and } \\
\text { empirical research } \\
\text { Aligning research design to research } \\
\text { problem } \\
\text { Research continuum: exploratory to } \\
\text { confirmatory }\end{array}$ \\
\hline $\begin{array}{l}\text { Course 2: The } \\
\text { single case } \\
\text { design }\end{array}$ & Low & $\begin{array}{l}\text { Case study and other designs within the } \\
\text { realms of a single case (e.g. experiments, } \\
\text { ethnography, action research, } \\
\text { phenomenological study) } \\
\text { Qualitative (open coding, categorizing and } \\
\text { relating; content analysis, method of } \\
\text { constant comparison, domain analysis, } \\
\text { taxonomic analysis, componential analysis, } \\
\text { process analysis) }\end{array}$ \\
\hline & & $\begin{array}{l}\text {-Interpretative/descriptive and } \\
\text { exploratory } \\
\text {-Explore descriptions of a } \\
\text { phenomenon to identify dimensions } \\
\text { and build a definition } \\
\text {-Explore antecedents and respective } \\
\text { processes leading to a phenomenon } \\
\text { to build an explanation }\end{array}$ & \\
\hline
\end{tabular}




\begin{tabular}{|l|l|l|l|}
\hline $\begin{array}{l}\text { Proposed } \\
\text { courses }\end{array}$ & $\begin{array}{l}\text { Level of } \\
\text { knowledge } \\
\text { from prior } \\
\text { research }\end{array}$ & Research Purpose & Learning Content/Outcomes \\
\hline & & & $\begin{array}{l}\text { Quantitative (Descriptive statistics when } \\
\text { unit of analysis is an organisation or } \\
\text { country, factor analysis, cluster analysis) }\end{array}$ \\
\hline $\begin{array}{l}\text { Course 3: } \\
\text { Small to } \\
\text { medium-N } \\
\text { case design }\end{array}$ & Medium & Explanatory & $\begin{array}{l}\text { Variable-centred research, } \\
\text { Mills methods, } \\
\text { Case-to-case transfer } \\
\text { Analytic generalizations } \\
\text { Qualitative comparative analysis (QCA) }\end{array}$ \\
\hline $\begin{array}{l}\text { Course 4: } \\
\text { Large-N case } \\
\text { design }\end{array}$ & High & Confirmatory & $\begin{array}{l}\text { Survey design and field experiments } \\
\text { Quantitative (univariate inferential } \\
\text { statistics, multivariate inferential statistics } \\
\text { Qualitative (axial coding) }\end{array}$ \\
\hline
\end{tabular}

\subsection{The Single Case}

Though the single case is best used to explore the meaning of a specific phenomenon whether at the individual, organizational or societal level, instructors can also use it to describe the process underlying a phenomenon, which can be used to identify actors, steps, conditions and variables to develop a tentative explanation. In this way, the single case can set up the building blocks for students to learn the explanatory logic required to understand large- $\mathrm{N}$ cross sectional or longitudinal surveys commonly associated with quantitative research. At this stage, all of this can be done with qualitative data. But quantitative data can be used to score the conditions identified from the process description as well as the resultant level of the outcome, be it low, high, weak, or strong. For example, for a case of organizational leadership, employee perception scores might be used to determine if the case is one of weak or strong leadership. Thus the irony of single case study, which is often left unspoken by instructors, is that unlike a large- $\mathrm{N}$ study, a single case can rely on a large number of observations to arrive at a "score" for a variable of interest; and reflections on correct measurement can be much more intensive (Blatter and Haverland, 2012).

We are already familiar with the usefulness of a single case for exploratory research. When we have little knowledge about a phenomenon, we use a single case to describe the essence of phenomenon. One can determine the meaning of the phenomenon as well its dimensions. This is important before proceeding to explain how the phenomenon has occurred. In addition, we might use multiple observations of the phenomenon to develop a quantitative type score to determine if the phenomenon is low, high, weak, or strong. For example, looking at the phenomenon of remuneration in a bank, we can determine by calculating a mean from a sample of employee salaries to locate the bank as a high or low paying employer. Because the human resources department has records of the entire population of employees, one can then also teach the idea of inference from the sample to the entire population. All of this-using both qualitative and quantitative data--fulfils a descriptive research objective. But the same case can be used to also fulfil an explanatory research objective.

If we wish to know how and why the phenomenon got to be low, high, weak, or strong, we can continue with the same case to fulfil an explanatory research objective. The initial descriptive research objective is important to agree on the meaning of the phenomenon we seek to explain. In explanatory research the same phenomenon serves as an outcome of a certain process in which conditions vary to give rise to changes in the outcome. Thus we can use the same case to identify the conditions that lead to an outcome of interest as well as to explore the process taken from a certain condition to the outcome. In this way, we explore how and why the outcome arises. This latter approach has been termed process tracing. Process tracing can be used to identify the actors and activities involved in certain events that lead to an outcome. Processing tracing can also be used to locate an $X$ condition. Evidence that an $X$ leads to a $Y$ can be sought in the sequence and structure of activities and/or in the testimony of actors about why they acted as they did (Van Evera, 2009). One way to make sense of this process approach is to look at it against a variance approach. Whereas the process approach looks at the actors and the activities leading to a certain outcome, a variance approach looks at a fixed condition that has varying attributes (see Van de Ven, 2007). In fact, the model I propose above about Xs and Zs leading to a Y has a bias towards this variance approach: it ignores the actors and activities involved in the process to get from $\mathrm{X}$ to $\mathrm{Y}$. 
We can use the same case to demonstrate inductive and deductive logic. We take an inductive approach and we use the case itself to identify causal factors and to trace how they are linked to the outcome. But one can also, in a deductive manner, develop a proposition about "what" the causal factor might be and conduct confirmatory research and extend this to investigate how and why the causal factor is linked to an outcome. If a student has propositions about certain antecedent conditions from the literature or their own experience, they can explore how a certain condition $(X)$ leads to an outcome $(Y)$. If within a particular case the value of $X$ is above a typical average value, then the value of $Y$ should also be above this average if the proposition were to hold (Van Evera, 2009).

For the latter, students develop concrete notions about variables and how a change in a causal factor, this time within a case over time instead of between cases, lead to a change in the outcome. This is an important building block for teaching regression for quantitative analysis: students can associate concrete changes in an outcome or dependent variable with changes in an antecedent condition or independent variable. In addition, studying the process from the causal factor to the outcome in a single case can also be used to introduce concepts such as mediation and moderation or the ideas of path analysis and contingency.

Notably, a single case can also be used to introduce an approximation of experimental logic through observing changes in an antecedent condition over time and any subsequent change in the outcome over the same time. This is also referred to as within-case analysis. This idea can also be used to convey the action research method where one can observe changes in an outcome of interest $(Y)$ after a deliberate intervention (X). Additionally, if a case is used to explore the customs and habits of a group of people, one can use this to convey the ethnographic method. If a case is used to explore the lived experiences of certain individuals, then one use this to convey the phenomenological method.

It is possible to demonstrate sophisticated statistical techniques like a multiple regression in a single case. For example in the case of an organization, one can regress employee data if one wishes to confirm whether certain individual-level phenomena, identified during the process tracing, is related to an individual outcome. But, one cannot conduct regression analyses for an organizational level outcome because of the lack of variation in the outcome, unless one does a longitudinal study. But I would suggest limiting the single case to demonstrate descriptive statistics (a mean salary, derived from an employee survey, for example).

The building blocks for quantitative data reduction techniques such as factor analysis and cluster analysis can also be introduced in a single case. For example, qualitative type thematic analysis also serves as a data reduction technique and can serve as a useful building block to teach the principles of data reduction applied in factor analysis and cluster analysis for large- $\mathrm{N}$ cases (Onwuegbuzie, 2003; Onwuegbuzie, Bustamante and Nelson, 2010b).

Ultimately, the single case is a useful design to demonstrate to students how to begin building the bare bones of a theory that includes the what, how, why, when, where and who. This involves students identifying the $X$ explanatory conditions and the $Z$ background conditions and beginning to trace how a single explanatory condition leads to a $\mathrm{Y}$ outcome. This latter exercise has been referred to as a within case approach and more recently as process tracing (Beach and Pederson, 2013). This exercise can lead to not only identifying certain conditions for an outcome phenomenon to occur but also to develop a theory about how and why a condition $X$ leads to an outcome $Y$.

\subsection{Small- $\mathrm{N}$ to medium- $\mathrm{N}$ Cases}

The small-N case design, also referred to as a multiple case study (Eisenhardt, 1989), provides the instructor an opportunity to introduce the logic of comparison to the student. Put another way, the small-N case design is conducive to demonstrating the building blocks of variance logic. This logic of comparison or variance is an important building block for students to understand qualitative comparative analysis or QCA (Ragin, 2014), which relies typically on Boolean analysis of a medium $\mathrm{N}$ cases. Ultimately, this foundation also helps students to make sense of the statistical methods used to compare differences in an outcome $(\mathrm{Y})$ and corresponding differences in a causal condition $(\mathrm{X})$ across large $\mathrm{N}$ cases typical in regression analysis.

According to variance logic one can observe the variance of a condition within the same case and between several cases. The logic of comparison is akin to between-case analyses in variance logic. It is founded on Mills methods of agreement and difference. The method of agreement tells us to look for conditions present on all 
occasions when an outcome of interest occurs. According to Mills, if the outcome of interest occurs in two cases, then we need to search amongst the conditions between the two cases until we locate one or more conditions either absent or present in both cases: the conditions must agree with one another. Thus if a researcher completes a single case study where an outcome of interest is present and decides to search for a second case with the same outcome also present, the researcher will attempt to locate conditions in the second case that were also present or absent in the first case. If the researcher selects a second case in which the outcome of interest is not present, then the researcher will search amongst the conditions between the two cases until he/she locates one that is present in one case and absent in the other. This corresponds to Mills method of difference, which tells us to look for some condition present on some occasion when the outcome of interest occurs and absent on an otherwise similar occasion when it does not. The condition or outcome need not always be assessed as present or absent; many times they can be assessed as weak, strong, low, or high; but it is useful to locate cases with large similarities or differences in outcome. The small- $\mathrm{N}$ case design is particularly conducive to Mills method of difference.

This approach to simply identifying conditions and using Millean logic has been termed deterministic as opposed to probabilistic. It has immense value in building theory. Once such research projects are done frequently and patterns begin to emerge then one can go onto spend resources to undertake a survey of many cases to enable probabilistic type inference. Otherwise it is often not necessary to proceed directly into a survey type design, which also comprises of cases but this time selected randomly within a sampling framework that has large differences in outcome levels; otherwise, it becomes difficult to detect variations.

Notably, the small-N case design is conducive to demonstrating the building blocks of variance logic. For starters one can use truth tables to demonstrate whether extreme variances (absence or presence) in certain $X$ conditions lead to similar changes in $Y$ conditions. For instance, to explore a theory that $X$ has a positive effect on $Y$ one needs to take two cases, say $A$ and $B$, if the value of $X$ is higher in case $A$ than $B$, the value of $Y$ should also be higher for case $A$ than case $B$. If the observations correspond to these expectations then the theory is gaining some ground in the real world. With more than one case, one can begin to introduce quantitative methods such as cross tabulation and analysis of variance. One can also introduce statistical techniques to compare means.

Finally, a small-N case design can also be used to teach the logic of purposive case selection against inductive or deductive logic. For deductive logic, where propositions are made about the presence of certain X conditions, cases are selected against these same conditions. For inductive logics, where the cases themselves are used to develop propositions, cases are selected against the absence or presence of the outcome.

The medium- $\mathrm{N}$ case design can be used to introduce several quantitative statistical concepts but is most frequently used to demonstrate the ideas behind QCA. QCA lays out cases in a truth table and then uses Boolean logic to locate configurations of antecedent conditions leading to either the presence or absence of an outcome of interest. At this stage, depending on the diverse contexts from which cases are drawn, one might still be fulfilling exploratory objectives. But if one is using Mills method of agreement, where one seeks agreement between certain antecedent conditions for a set of diverse cases with similar outcomes, one may use QCA to fulfil confirmatory research objectives. Typically, QCA is designed around Mills method of agreement.

\subsection{Large-N Cases}

The large- $\mathrm{N}$ case design is synonymous with quantitative probabilistic techniques. It can be used to demonstrate both descriptive and inferential statistics. For instance, it can be used to describe the data gathered to measure a condition in terms of its normality. It can also be used to demonstrate inferences through panel analysis, structural equational modelling and a variety of regression techniques e.g. Probit and Logit regressions.

But by the time the instructor gets to this segment of research methodology, one can expect the student to have a sound understanding of the underlying variance approach from the earlier course on small to medium-N case design. Only this time, instead of relying on truth tables, the instructor demonstrates with statistical probability concepts that the variances in an outcome can be isolated to variances in certain antecedent conditions. Thus the teaching of the design and analysis of small to medium- $\mathrm{N}$ cases before large- $\mathrm{N}$ cases offers a promising way to demonstrate the underlying logic of quantitative statistical techniques to students who experience difficulty with learning statistics (compare with Onwuegbuzie et al, 2010a). 
Note that the phenomenon of interest and respective cases for a large- $\mathrm{N}$ case designs cannot be aligned to an outcome phenomenon because selection on the dependent variable is not advisable when the research purpose is to confirm that a theory applies to a broader population. One cannot make an inference based on a sample restricted to a particular outcome of a phenomenon. The sampling framework of cases can be based on a certain causal condition that emerged in studying a single case or a small to medium number of cases. The cases are then selected randomly from this sampling framework.

\section{Discussion and Conclusions}

This paper contributes to the discourse about research methodology pedagogy for developing new scholarly researchers (Christ, 2009; Earley, 2007; Hesse-Biber, 2015; Ivankova and Clark, 2018; Onwuegbuzie et al, 2013; Tashakkori and Teddlie, 2010). It proposes that we teach research methodology around an overall explanatory research purpose and around the extent of prior knowledge about a phenomenon (Edmondson and McManus, 2007). In this schema, descriptive research is a building block to this broader explanatory purpose (de Vaus, 2001). For new phenomena, this explanatory purpose is met by navigating through an exploratory-confirmatory research continuum (Onwuegbuzie and Leech, 2005). Along this continuum, the "case" serves as the underlying analytical unit. The case can represent behaviour at an individual, firm, group or societal level. The case can also be an instantiation of an intervention as in action research; or an instantiation of group behaviour as in an ethnography.

To help the student navigate the exploratory-confirmatory research continuum, I propose an N-case approach for designing and teaching research methodology courses. The $\mathrm{N}$ represents the number of cases required by instructors to illustrate various research methods. I suggest an order where instructors begin with a single case to demonstrate how new insights emerge before moving on to demonstrate to students how to confirm these insights more generally by applying statistical methods to a large number of cases. Thus after an introductory course on the theory building process, I propose that the second course be designed around a single "case" to show students how scholars explore an explanation for a phenomenon of interest. Then, in a third course, instructors can teach students how the emerging explanation is developed and even challenged through case comparisons. Accordingly, this third course relies on small to medium- $\mathrm{N}$ cases. Ultimately the fourth and final course relies on large- $\mathrm{N}$ cases to teach students how to confirm a theory with inferential and probabilistic type statistics. The final course can also include a lesson on longitudinal panel type probabilistic analytical techniques. Panel data analysis, for example, involves making use of both information from changes over time within the same case as well as information from differences between cases. Thus a research methodology module can begin with a single case, moving to small-N cases, then to a medium- $\mathrm{N}$ cases and finally to a large- $\mathrm{N}$ probabilistic type study. This teaching framework differs from many current research teaching frameworks: it treats qualitative and quantitative categories not as distinct research strategies, but as distinct types of data: the former features textual data while the latter features numerical data. Furthermore, it delays emphasis on statistical methods to much later in the research methods curriculum when students have grasped the underlying process in which certain antecedent conditions lead to an outcome.

One can also view this exploratory-confirmatory continuum as a move from studying the particular to the general. At a basic level, this involves moving from a within-case analysis of a single case to a between-case analysis of at least two cases and eventually, for a more complete explanation, teaching students the importance of relying on insights from both within and between case information. In this way, instructors can also demonstrate descriptive research goals to students: they can show students how they can use a single case to translate a phenomenon into an existing or new concept, and how more cases can be added to test the robustness of this conceptual representation. Along this continuum, Onwuegbuzie et al (2010a) have shown how instructors can apply both qualitative and quantitative analytical techniques. Borrowing from them, I show how instructors can scaffold the teaching of qualitative and quantitative analytical techniques along this continuum.

Also, when faced with a new phenomenon it is important to first describe it before explaining it (de Vaus, 2001). It is important to be sure about the facts and dimensions of the phenomenon. If the basic premise about the nature and existence of a certain phenomenon is incorrect, then attempts to explain a non-existent phenomenon become silly. This is not to say that a single case study can be used only to fulfil a descriptive purpose. Case studies can also be used to trace the process leading to an outcome phenomenon and thus help form the initial attempts at building an explanation. 
Notably, prevailing teaching practices tends to move in the opposite direction from large-N designs to a single case (e.g. see Mahoney, 2008). A major implication of the teaching approach I propose is to replace the focus on types of data-qualitative versus quantitative-with one that focuses on the number of cases required to meet specific research objectives, be they exploratory and/or confirmatory or descriptive and/or explanatory. The irony of single case study, which is often left unspoken by instructors, is that unlike a large- $\mathrm{N}$ design, which relies on a single observation per case, a single case relies on a large number of observations to arrive at a "score" for a variable of interest; and reflections on correct measurement are much more intensive (Blatter and Haverland, 2012). Thus, a single case can often rely on both qualitative and quantitative types of data. The underlying rationale here is that qualitative and quantitative research can be compatible; both can underpin the same logic; and both can contribute towards the same research objective (Petticrew and Roberts, 2008).

The N-case approach also implies that we need not advocate to students that they commit themselves to a paradigm: say positivism versus interpretivism. Neither should they choose between qualitative and quantitative approaches even before they are ready to formulate a research question. With current approaches to teaching research methodology, we risk young researchers developing identities as either qualitative or quantitative researchers. For example, if they are hesitant to work with numbers, they will lean towards qualitative approaches to answer their research questions (Gorard, 2010). This means they set exploratory or interpretative research purposes despite the rich prior empirical knowledge available about the phenomenon where one is better off deducing a hypothesis and using probabilistic statistical models to confirm it across a large number of cases located in a setting not examined before (Edmondson and McManus, 2007). When we are closer to a description and explanation that has been developed by other scholars, there is little need to return to interpretative type research designs unless one needs to challenge the original interpretation and resultant explanation of the phenomenon. In this respect, it becomes important to reflect on whether research design pedagogy has recognized the need to develop new researchers that can match their research designs to the extent of knowledge available about a phenomenon. It is important then to teach students to devise whatever methods appropriate for finding a solution to the research problem at hand. Paradigms can then be brought into line with a method or a combination of methods. This argument deserves further attention and scholars might consider interrogating this line of argument.

Notably, this approach to teaching might overcome certain ethical implications of researchers limiting themselves to either qualitative or quantitative research. For example, those researchers who use funds sourced from public tax income must be concerned about the robustness of their findings and the validity of their conclusions (Gorard, 2010). If publically funded researchers are hesitant to work with numbers, they risk limiting their research designs to those that rely on qualitative data and ignoring essential forms of quantitative evidence. Ultimately, it is not advisable to design research to apply ones favoured method without attention to the questions one seeks to answer (de Vaus, 2001; Gorard, 2010; Sandelowski, 2014).

The approach outlined in this paper is not without limitations. The term "case", particularly in the context of small to large-N cases, is used conveniently here. Specifically, I have use the word case/s as a synonym for the unit/s being analysed. But though the "case" may take the form of a unit of analysis in the approach described here, typically a case is an instantiation of a certain phenomenon (e.g. Gerring, 2007). In other words, it is not a "case" when the phenomenon is absent. But a brief read across the World Wide Web sees the case study often being referred to as a descriptive and exploratory analysis of a person, group or event and yet others indicate that it must be further confined to an instantiation of a certain phenomenon. I've considered a case to be a representative of all of these. In addition, my use of the term "case" might be considered for its convenience in illustrating how an explanation of a certain phenomenon can be developed along an exploratory-confirmatory continuum. However, when one teaches probabilistic methods, one must caution students about sample selection limited only to the occurrence of an outcome phenomenon. Sampling frameworks are better developed from the presence of antecedent conditions. For example, entrepreneurship scholars caution against selecting only entrepreneurs when the studying the phenomenon of choosing to start one's own business over seeking paid employment (Davidsson and Gordon, 2012).

To conclude, this paper has argued that instructors use a case-oriented approach to emphasise the "case" as the basic pedagogic unit to scaffold the student's learning of research methodology. Rather than delineating research methodology curricula into quantitative and qualitative designs and methods, I advocate delineating them into single, small-to-medium-N and large-N case designs. This calls for an approach that displaces a focus on types of data-qualitative versus quantitative- with one that focuses on the number of cases required to 
meet specific research objectives, be they exploratory and/or confirmatory. Evidently, this bottom-up approach of building a causal argument has yet to reach our research methodology pedagogy.

Instructors are encouraged to move away from introducing their teaching of causal models with inferential statistical techniques. Instead, they are encouraged to teach research methodology by developing a substantive theory from observing causal processes at the level of a particular case and then using this understanding as a building block to explain how the theory is more generally applicable to a large number of cases. Students will find it simpler to develop a mental picture of co-variational observations from single or small $\mathrm{N}$ cases and this can serve as a useful building block to understand the co-variational thinking applied to complex statistical techniques used for large- $\mathrm{N}$ designs. Meanwhile, let us increase our dialogue about to how to implement these ideas in a research methodology class. For starters, we can benefit from action research around the $\mathrm{N}$-case approach.

\section{References}

Beach, D., and Pedersen, R. 2013. Process-tracing methods: foundations and guidelines. Ann Arbor, MI: University of Michigan Press.

Blatter, J., and Haverland, M. 2012. Designing case studies: explanatory approaches in small-N research. Houndmills Basingstoke: Palgrave Macmillan.

Christ, T.W., 2009. Designing, teaching, and evaluating two complementary mixed methods research courses. Journal of Mixed Methods Research, 3(4), pp.292-325.

Davidsson, P. and Gordon, S.R., 2012. Panel studies of new venture creation: a methods-focused review and suggestions for future research. Small Business Economics, 39(4), pp.853-876.

De Vaus, D.A., 2001. Research Design in Social Research. Sage: London

Earley, M.A., 2007. Developing a syllabus for a mixed-methods research course. International Journal of Social Research Methodology, 10(2), pp.145-162.

Edmondson, A.C. and McManus, S.E., 2007. Methodological fit in management field research. Academy of Management Review, 32(4), pp.1246-1264.

Eisenhardt, K. M. 1989. Building theories from case study research. Academy of Management Review, 14(4), pp.532-550.

Gerring, J. 2007. Case study research: principles and practices. Cambridge: Cambridge University Press.

Gorard, S. 2010. Research design, as independent of methods. In: A. Tashakkori and C. Teddlie, (eds). Handbook of mixed methods in social and behavioral research. Thousand Oaks, CA: Sage. pp.237-252.

Hall, P. A. 2003. Aligning ontology and methodology in comparative research. In: J. Mahoney and D. Rueschemeyer, (eds). Comparative historical analysis in the Social Sciences. New York: Cambridge University Press. pp.373-404.

Hesse-Biber, S., 2015. The problems and prospects in the teaching of mixed methods research. International Journal of Social Research Methodology, 18(5), pp.463-477.

Ivankova, N.V. and Plano Clark, V.L., 2018. Teaching mixed methods research: using a socio-ecological framework as a pedagogical approach for addressing the complexity of the field. International Journal of Social Research Methodology, 21(4), pp.409-424.

King, G., Keohane, R. O., and Verba, S. 2007. Designing social inquiry: scientific inference in qualitative research. Princeton, NJ: Princeton University Press

Mahoney, J. 2008. Toward a unified theory of causality. Comparative Political Studies, 41(4-5), pp.412-436.

Nind, M. and Lewthwaite, S., 2019. A conceptual-empirical typology of social science research methods pedagogy. Research Papers in Education, pp.1-21.

Onwuegbuzie, A.J., 2003. Effect sizes in qualitative research: a prolegomenon. Quality and Quantity, 37(4), pp.393-409.

Onwuegbuzie, A. J., and Leech, N. L. 2005. On becoming a pragmatic researcher: the importance of combining quantitative and qualitative research methodologies. International Journal of Social Research Methodology, 8(5), pp.375-387.

Onwuegbuzie, A.J., Leech, N.L., Murtonen, M. and Tähtinen, J., 2010a. Utilizing mixed methods in teaching environments to reduce statistics anxiety. International Journal of Multiple Research Approaches, 4(1), pp.28-39.

Onwuegbuzie, A.J., Bustamante, R.M. and Nelson, J.A., 2010b. Mixed research as a tool for developing quantitative instruments. Journal of Mixed MethodsRresearch, 4(1), pp.56-78. 
Onwuegbuzie, A.J., Frels, R.K., Collins, K.M. and Leech, N.L., 2013. Conclusion: a four-phase model for teaching and learning mixed research. International Journal of Multiple Research Approaches, 7(1), pp.133-156.

Petticrew, M. and Roberts, H., 2008. Systematic reviews in the social sciences: a practical guide. New York: John Wiley \& Sons.

Ragin, C. 2014. The comparative method: moving beyond qualitative and quantitative strategies. Oakland University of California Press.

Sandelowski, M. 2010. What's in a name? Qualitative description revisited. Research in Nursing and Health, 33(1), pp.77-84.

Sandelowski, M. 2014. Unmixing mixed-methods research. Research in Nursing and Health, 37(1), pp.3-8.

Tashakkori, A., and Teddlie, C. 2010. Handbook of mixed methods in social and behavioral research. Thousand Oaks, CA: Sage.

Van de Ven, A. H. 2007. Engaged scholarship: a guide for organizational and social research. Oxford: Oxford University Press.

Van Evera. S. 2009. Guide to methods for students of political science. Ithaca, NY: Cornell University Press.

Whetten, D. A. 1989. What constitutes a theoretical contribution? Academy of Management Review, 14(4), pp.490-495.

Yin, R.K., 1984. Case study research: design and methods. Beverly Hills, California: Sage Publications. 